Check for updates

Cite this: RSC Adv., 2017, 7, 45960

Received 15th August 2017

Accepted 22nd September 2017

DOI: 10.1039/c7ra09012a

rsc.li/rsc-advances

\section{Binding behaviors and structural characteristics of ternary complexes of $\beta$-lactoglobulin, curcumin, and fatty acids $\uparrow$}

\author{
Jingwen Liu, ${ }^{\text {ab }}$ Lun Jiang, ${ }^{\text {ab }}$ Yunyue Zhang, ${ }^{\text {ab }}$ Zhongyao Du, ${ }^{\text {ab }}$ Xiaoxia Qiu, ${ }^{\text {ab }}$ \\ Lingyan Kong ${ }^{\mathrm{C}}$ and Hao Zhang (iD)*ab
}

\begin{abstract}
In this study, we evaluated the binding behaviors and structural characteristics of multi-ligand complex of $\beta$ lactoglobulin $(\beta-\mathrm{lg})$ with curcumin and fatty acids. Methods of fluorescence spectroscopy, circular dichroism spectroscopy, dynamic light scattering, Turbiscan stability analysis, HPLC quantification, molecular docking studies, and molecular dynamics simulation were used. The results of fluorescence measurements indicated that $\beta$-lg was able to interact with curcumin and fatty acids at the same time, and the presence of fatty acids enhanced the binding constant of curcumin. The formation of ternary complexes with curcumin and fatty acids did not change the secondary structure of $\beta$-lg, while this process resulted in a considerable increase in particle size and negative charges compared to the native $\beta$-lg (ending up at around $145 \mathrm{~nm}$ and $-30 \mathrm{mV}$ in the ternary complexes). The loading efficiencies of the fatty acids were $32 \pm 2 \%$ in $\beta$-lg-myristic acid (C14:0)-curcumin and $34 \pm 3 \%$ in $\beta$-lg-palmitic acid (C16:0)-curcumin, and those of curcumin were $58 \pm 2 \%$ in $\beta$-lg-C14:0-curcumin and $57 \pm 2 \%$ in $\beta$-lgC16:0-curcumin. These findings provide basic information to clarify the binding mechanism for the interactions of $\beta$-lg with ligands at multiple sites on the molecular level.
\end{abstract}

\section{Introduction}

In recent years, there has been a growing interest in the food industry to incorporate various bioactive ingredients due to their health benefits. Among them, curcumin (diferuloylmethane), a natural polyphenolic compound derived from the herb turmeric (Curcuma longa), is known to exhibit numerous biological and pharmacological activities, such as anti-tumor, anti-oxidative, and anti-inflammatory properties. ${ }^{\mathbf{1 , 2}}$ In addition, several clinical trials showed that curcumin is not toxic to humans, even at high doses (up to $8 \mathrm{~g}$ per day for 3 months). ${ }^{3}$ Therefore, curcumin is regarded as a promising nutraceutical to be used in functional foods. However, being a hydrophobic molecule, the poor water solubility and bioaccessibility of curcumin limit its application and biological activities. To enhance the solubility and bioavailability of curcumin, a number of

${ }^{a}$ Beijing Advanced Innovation Center for Food Nutrition and Human Health, College of Food Science and Nutritional Engineering, China Agricultural University, Beijing 100083, P. R. China. E-mail: zhanghaocau@cau.edu.cn; Fax: +86-10-62736344; Tel: +86-10-62736344

${ }^{b}$ Key Laboratory of Functional Dairy, Co-constructed by Ministry of Education and Beijing Government, Beijing Laboratory of Food Quality and Safety, China Agricultural University, Beijing 100083, P. R. China

${ }^{c}$ Department of Human Nutrition \& Hospitality Management, The University of Alabama, Tuscaloosa 35487, AL, USA

$\dagger$ Electronic supplementary information (ESI) available. See DOI: 10.1039/c7ra09012a researchers suggested complexation of curcumin with bio-based polymeric ingredients, e.g. albumin, phospholipid, casein micelles, chitosan, and modified starches. ${ }^{\mathbf{4}-8}$

Milk protein-based nanoparticles are promising delivery vehicles for nutraceuticals because milk proteins have naturally evolved to deliver nutrients from mother to neonate. Bovine $\beta$ lactoglobulin $(\beta-\mathrm{lg})$ is the major whey protein from bovine milk. Bovine $\beta$-lg consists of a 162 amino acid-long mature polypeptide with a molecular weight of approximately $18.3 \mathrm{kDa} .^{9}$ It has a hydrophobic core, containing eight antiparallel $\beta$-strands $(\mathrm{A}-\mathrm{H})$, which is called the calyx and is the main binding site of the protein. Furthermore, $\beta$-lg is conditional resistant to acidity at low $\mathrm{pH}$ of stomach, and to digestion by gastric proteases. ${ }^{\mathbf{1 0 , 1 1}}$ Therefore, it is an attractive candidate to serve as an encapsulant for delivering hydrophobic nutrients and improving their bioavailability. For example, $\beta$-lg-based delivery strategies have been developed to carry vitamin $\mathrm{D}$ and naringenin to improve their bioavailability. ${ }^{\mathbf{1 2 , 1 3}}$ Moreover, a number of studies demonstrated that the binding of curcumin to $\beta$-lg could enhance the solubility and antioxidant activity of curcumin. ${ }^{\mathbf{1 4 , 1 5}}$

However, most of the studies have focused on transporting single ligand or the interferences and competitions of secondary ligand to protein-monoligand complex. ${ }^{16,17}$ In fact, the trend to incorporate different bioactive compounds into food formulations and offer multiple benefits simultaneously has increased remarkably. ${ }^{18}$ It is thus required to develop carriers that can bind a plurality of bioactive components at the 
same time. $\beta$-lg has more than one site for ligand binding, with the main site in the calyx and other possible sites at the dimer interface, the outer surface near Trp19-Arg124, and the hydrophobic pocket in a groove between the $\alpha$-helix and the $\beta$ barrel. ${ }^{19-22}$ Hence, we speculated that $\beta$-lg could be used as a carrier for more than one type of hydrophobic molecule at a time. However, the specific binding behaviors of $\beta$-lg delivering multiple ligands simultaneously are still not clear. To this end, three fatty acids, i.e. caprylic acid (C8:0), myristic acid (C14:0), and palmitic acid (C16:0), were selected as the second type of hydrophobic molecule to form ternary complexes with $\beta$ $\lg$ and curcumin. Previous studies noted that $\beta$-lg has different affinities to fatty acids of different structures. ${ }^{23}$ Therefore, the binding of curcumin to $\beta$-lg may be affected by different fatty acids, and vice versa.

The main objective of this study was to clarify the binding mechanism and structural characteristics of ternary complexes of $\beta$-lg, curcumin, and fatty acids. Complimentary techniques, i.e. fluorescence spectroscopy, circular dichroism (CD) spectroscopy, dynamic light scattering (DLS), Turbiscan stability analysis, loading efficiencies measurements, molecular docking studies, and molecular dynamics (MD) simulation were applied to measure the binding kinetics and probe the structural details of the complexes. This study provides valuable information for developing new $\beta$-lg-based delivery systems for multiple ligands with applications in the food industry.

\section{Materials and methods}

\section{Materials}

Bovine $\beta$-lg (purity $>90 \%$ ) was purchased from Sigma-Aldrich Chemical Co. (St. Louis, Missouri). Curcumin $(\log P=3.6$, purity $>98 \%$ ) was obtained from J\&K chemical (Shanghai, China). C8:0 $(\log P=2.9$, purity $>98 \%)$, C14:0 $(\log P=6.1$, purity $>99 \%$ ), and C16:0 ( $\log P=7.2$, purity $>97 \%$ ) were purchased from Tokyo Chemical Industry (Tokyo, Japan). Other reagents were of analytical grade, and double distilled water was used throughout.

\section{Preparation of $\boldsymbol{\beta}$-lg, curcumin, and fatty acids complexes}

To obtain a protein solution, $\beta$-lg was dissolved in an aqueous solution of $10 \mathrm{mM}$ Tris-HCl buffer ( $\mathrm{pH}$ 7.4). The protein concentration was determined using a molar absorption coefficient of $\varepsilon_{278 \mathrm{~nm}}=17600 \mathrm{M}^{-1} \mathrm{~cm}^{-1}$ on a UV-vis spectrophotometer. ${ }^{24}$ Fresh curcumin solution $(4 \mathrm{mM})$ was prepared by dissolving it in ethanol, and the solutions were protected from light throughout the experiments. The exact concentration of curcumin was measured using a molar absorption coefficient of $\varepsilon_{429 \mathrm{~nm}}=55000 \mathrm{M}^{-1} \mathrm{~cm}^{-1} \cdot{ }^{25}$ Ethanol solutions of C8:0, C14:0, and C16:0 (1 mM) were also prepared in advance.

The above solutions were diluted in Tris- $\mathrm{HCl}$ to various concentrations required for further preparing the complex samples. The complex samples were prepared by mixing the diluted solutions in varying proportions. The resulting ethanol concentration never exceeded $10 \%(\mathrm{v} / \mathrm{v})$, and this concentration had no appreciable effect on protein structure. ${ }^{\mathbf{1 6}}$

\section{Fluorescence measurements}

To reveal the binding behaviors between $\beta-\lg$ and ligands, changes of $\beta$-lg tryptophan (Trp) fluorescence and curcumin fluorescence emission intensity were measured. First, curcumin $(0-50 \mu \mathrm{M})$ was added into the $\beta$-lg solution and the $\beta$-lg-fatty acid complexes, in which the concentrations of $\beta$-lg and fatty acid were $2 \mu \mathrm{M}$ and $300 \mu \mathrm{M}$, respectively. Moreover, in order to clarify the effect of fatty acids on the binding kinetics between curcumin and $\beta$-lg, the binding constants, $K_{\mathrm{a}}$, were calculated according to the fluorescence intensities of $\beta$-lg. In this case, the concentrations of curcumin were $0,1.6,6,10,16,30,50,60,80,100,150,200 \mu \mathrm{M}$. Secondly, to assess the effect of curcumin presence on the binding between fatty acids and $\beta$-lg, each fatty acid $(0-200 \mu \mathrm{M})$ was added into the $\beta$-lg solution $(2 \mu \mathrm{M})$ and the $\beta$-lg-curcumin complex containing $2 \mu \mathrm{M}$ of $\beta$-lg and $150 \mu \mathrm{M}$ of curcumin.

Fluorescence measurements were performed on a CARY Eclipse spectrofluorometer (Varian, Palo Alto, California). Binding of these ligands was evaluated using the fluorescence emission quenching of $\beta$-lg $\operatorname{Trp}\left(\lambda_{\mathrm{ex}}=295 \mathrm{~nm}, \lambda_{\mathrm{em}}=330 \mathrm{~nm}\right)$ and the change in fluorescence emission intensity for curcumin $\left(\lambda_{\mathrm{ex}}=425 \mathrm{~nm}, \lambda_{\mathrm{em}}=500 \mathrm{~nm}\right)$. The fluorescence intensities were shown as the average of three replicates. Because some ligands possess intrinsic fluorescence, the intensities of $\beta$-lg and curcumin in the samples were automatically subtracted from the emission intensities of the corresponding solutions. In addition, some ligands are able to absorb energy both at the excitation wavelength and emission wavelength, which is known as the inner-filter effect. Therefore, the measured fluorescence emission intensity was corrected according to eqn (1): ${ }^{26}$

$$
F_{\mathrm{c}}=F_{\mathrm{m}} \times 10^{\frac{A_{\mathrm{ex}}+A_{\mathrm{em}}}{2}}
$$

where $F_{\mathrm{c}}$ is the corrected fluorescence intensity; $F_{\mathrm{m}}$ is the measured fluorescence intensity; $A_{\mathrm{ex}}$ and $A_{\mathrm{em}}$ are the absorbance of the system at the excitation and emission wavelengths, respectively.

\section{CD measurements}

CD spectra were recorded using a CD spectropolarimeter (Pistar $\pi$-180, Applied Photophysics Ltd., Surrey, U.K.). The concentration of $\beta$ - $\lg$ was $10 \mu \mathrm{M}$ for the far UV region (190-260 $\mathrm{nm}$ ). The molar ratio of $\beta$-lg, curcumin, and fatty acids was $1: 1: 1$ in the ternary complexes. The path length was $0.1 \mathrm{~cm}$. Ellipticity was recorded at a speed of $100 \mathrm{~nm} \mathrm{~min}{ }^{-1}, 0.2 \mathrm{~nm}$ resolution, 20 accumulations, and $2.0 \mathrm{~nm}$ bandwidth. The collected data were analyzed on Dichroweb (http://dichroweb.cryst.bbk.ac.uk). The proportions of $\alpha$-helix, $\beta$-sheet, turn, and unordered coil were estimated using the SELCON3 program. ${ }^{27}$ The buffer background was subtracted from the raw spectra, and each spectrum was obtained as the average of three consecutive scans.

\section{DLS measurements and stability analysis}

The concentrations of $\beta$-lg, curcumin, and fatty acids were 200 $\mu \mathrm{M}$ in the $\beta$-lg-curcumin, $\beta$-lg-fatty acids, and the ternary complexes. For DLS measurements, the samples $(1.5 \mathrm{~mL})$ were analyzed at $25{ }^{\circ} \mathrm{C}$ with a Zetasizer Nano-ZS90 (Malvern 
Instruments, Worcestershire, U.K.). The intensity of light scattered was monitored at $173^{\circ}$ for size measurements. The results were recorded as the cumulant mean diameter (nm) and polydispersity index for particle size and size distribution, respectively. All experiments were performed in triplicates. For the zeta potential, the values reported are the average of measurements for three freshly prepared samples, and three readings were recorded for each sample.

The stability of the ternary complexes at $4{ }^{\circ} \mathrm{C}$ during 14 days storage were monitored by two methods, DLS measurement and Turbiscan analysis. The particle sizes, zeta potentials and Turbiscan curves were recorded at 1, 2, 3, 4, 5, 6, 7, 10, 12, and $14 \mathrm{~d}$. The Turbiscan stability analysis was determined using Turbiscan LAB (Formulaction, Toulouse, France). The Turbiscan scanned the samples from the bottom to the top with near infrared light $(\lambda=880 \mathrm{~nm})$ and measured the percentage of transmission through the samples as a function of the height of the tube, in order to quantify the rate of destabilisation.

\section{Loading efficiencies of curcumin and fatty acids}

To assess the loading efficiencies of curcumin and fatty acids within the complexes, $500 \mu \mathrm{L} \beta$-lg-curcumin, $\beta$-lg-fatty acids, and the ternary complexes were prepared. The concentrations of $\beta$-lg, curcumin, and fatty acids were $37.5 \mu \mathrm{M}, 75 \mu \mathrm{M}$, and 75 $\mu \mathrm{M}$, respectively. The samples were transferred into Amicon Ultra centrifugal filter devices with a molecular weight cutoff of $10 \mathrm{kDa}$ (Millipore, Boston, MA). The curcumin and fatty acids bound to $\beta$-lg were separated from the free ligands by centrifugal ultrafiltration $(14000 \times g, 1 \mathrm{~h})$. The retentate above the membrance was removed. After this, the ligands remaining in the membrance were collected by running another washing step based on the method described in previous study. ${ }^{28}$ The free ligands in the both two permeates were analyzed by HPLC. For reference samples, curcumin and fatty acids dissolved in ethanol were added to Tris-HCl solutions at the same $\mathrm{pH}$ and composition as that used for the complexes, but without the protein. Before this, an experiment was performed to confirm that after an additional washing step, the vast majority of the free ligands could be collected. The loading efficiency was calculated using the following equation: ${ }^{28}$

$$
\begin{aligned}
& \text { Loading efficiency }(\%) \\
& \qquad=\frac{(\text { total amount of ligands }- \text { free ligands })}{\text { total amount of ligands }} \times 100
\end{aligned}
$$

The amounts of curcumin and fatty acids were quantified using a Shimadzu LC-20AT HPLC instrument (Kyoto, Japan). The analysis was performed on a C18 HPLC column, with UV detection at $425 \mathrm{~nm}$ for curcumin and $310 \mathrm{~nm}$ for the fatty acids after derivatization. Fatty acids $(50 \mu \mathrm{L})$ were directly derivatized by treatment with 2-nitrophenylhydrazine hydrochloride $(100 \mu \mathrm{L})$ in the presence of $200 \mu \mathrm{L}$ of 1-(3-dimethylaminopropyl)-3-ethylcarbodiimide hydrochloride, to obtain non-volatile hydrazine derivatives. The derivatized fatty acids were separated from other acid hydrazides and interfering components by a solvent extraction using $1.5 \mathrm{~mL}$ of $n$-hexane.
After that, the samples were treated under a stream of nitrogen gas, and the residues were dissolved in methanol. The mobile phase was a mixture of water (A) and acetonitrile (B) at flow rate of $0.8 \mathrm{~mL}^{-1} \mathrm{~min}^{-1}$, and acetic acid $(0.12 \% \mathrm{v} / \mathrm{v})$ was added to the mobile phase. For curcumin, the injection volume was $100 \mu \mathrm{L}$, and the gradient elution program was as follows: 0-9 min, $44 \% \mathrm{~B}$ to $62 \% \mathrm{~B}$; $9-30 \mathrm{~min}, 62 \% \mathrm{~B}$. For the derivatized fatty acids, the injection volume was $40 \mu \mathrm{L}$, and the gradient elution condition was as follows: 0-5 $\mathrm{min}, 75 \% \mathrm{~B}$; 5-30 min, 95\% B. Four replicates were made to construct the calibration curve with curcumin and fatty acids standard solutions, ranging from 0 to $100 \mu \mathrm{M}$.

\section{Molecular docking studies and MD simulation}

The three dimension structure of $\beta-\lg$ was downloaded from RCSB Protein Data Bank (http://www.rcsb.org) with the ID 2Q2M. The structure of curcumin, C14:0, and C16:0 were obtained from NCBI with the PubChem compound CIDs of 969516, 11005, and 985, respectively. Binding interactions between ligands and protein were predicted by molecular docking method using AutoDock 4.0 software. All water molecules were removed and polar hydrogens were added at the beginning of the docking experiment. The docking experiment was performed on the whole protein. To recognize binding sites in the protein, the grid center was set at $80 \AA$, $80 \AA$, $80 \AA$. For more accurate docking, the optimized AutoDocking parameters were as follows: the maximum number of energy evaluations were 25000000 per run; the iterations of Solis and Wets local search were 3000 ; the number of generations was 100 , and the number of individuals in population was 300. All other parameters were kept as default values. Result differing by less than $2 \AA$ in a positional root mean square deviation (RMSD) were clustered together. The results of molecular docking were shown by Discovery Studio 4.0 (Accelrys, San Diego, California) to understand the binding interactions and get the lowest energy conformations of the obtained complexes. To investigate the stability of ligand-protein complex, MD simulation was also used. The most favorable ligand-protein complex obtained from docking calculations was regarded as the initial geometry for MD via Materials Studio Version 8.0 (Accelrys, San Diego, California). All MD simulations were performed by using the Amorphous Cell and Forcite module of the Material Studio simulation package with COMPASS. In the MD simulation, first, the amorphous cell module was employed to construct a box, which was inundated with 200 water molecules and the ligandprotein complex. Then, to equilibrate the system, the solute was subjected to the position-restrained dynamics simulation of NPT at $300 \mathrm{~K}$ for $20 \mathrm{~ns}$. The RMSD of a selected element can be calculated by least-squares fitting the structure to the reference structure. The results were displayed in Discovery Studio 3.0 (Accelrys, San Diego, California).

\section{Statistical analysis}

All of the data were analyzed using SPSS 17.0 package (SPSS Inc., Chicago, Illinois) and are expressed as means \pm standard deviations. One-way analysis of variance was used to estimate the statistical differences. Significant differences $(p<0.05)$ 
between means were determined using Duncan's multiple range tests.

\section{Results and discussion}

\section{Fluorescence measurements}

Fluorescence spectroscopy was used to monitor the intermolecular binding behavior of $\beta$-lg and curcumin, both of which produce fluorescence emission upon different excitation wavelengths. Binding of $\beta-\lg$ and curcumin will change the polarity of the surrounding environment of the fluorophores, and thus affect their fluorescence intensities. At a fixed curcumin concentration $(2 \mu \mathrm{M})$, the fluorescence intensity of curcumin increased with increasing $\beta-\lg$ concentration (Fig. S1 $\dagger$ ). Apparently, with the addition of $\beta-\mathrm{lg}$, curcumin was transferred from a relatively more hydrophilic aqueous environment to a more hydrophobic environment, i.e. the binding sites on $\beta$-lg.

When curcumin was gradually added to the $\beta$-lg and $\beta$-lgfatty acids complexes, a decrease in $\beta$-lg fluorescence intensity at $330 \mathrm{~nm}$ and a concurrent increase in curcumin fluorescence emission intensity at $500 \mathrm{~nm}$ were observed (Fig. 1). It indicated that curcumin bound to $\beta$-lg despite the presence of fatty acids. Furthermore, the binding constants of curcumin to $\beta$-lg in the absence and in the presence of fatty acids were calculated. To this end, the fluorescence intensities data were analyzed according to the following equation: ${ }^{26}$

$$
\frac{F_{0}-F}{F_{0}-F_{\mathrm{C}}}=\frac{[\mathrm{P}]_{\mathrm{t}}+[\mathrm{Q}]_{\mathrm{a}}+K_{\mathrm{d}}-\sqrt{\left([\mathrm{P}]_{\mathrm{t}}+[\mathrm{Q}]_{\mathrm{a}}+K_{\mathrm{d}}\right)^{2}-4 \times[\mathrm{P}]_{\mathrm{t}} \times[\mathrm{Q}]_{\mathrm{a}}}}{2 \times[\mathrm{P}]_{\mathrm{t}}}
$$
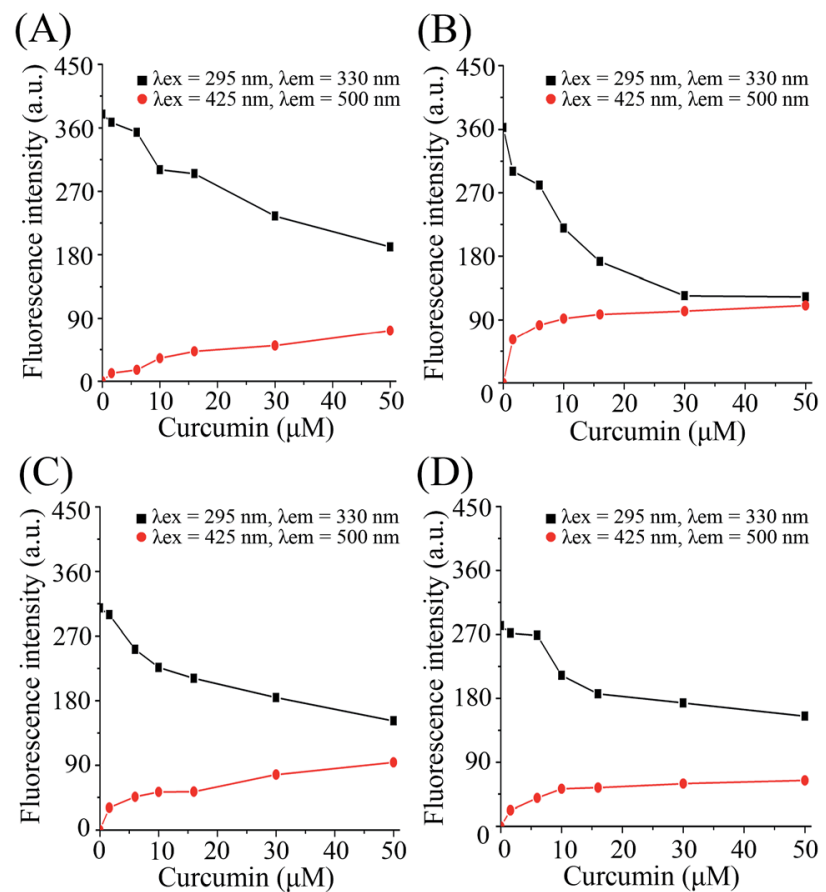

Fig. 1 Fluorescence emission intensity with addition of curcumin to $\beta$ $\mathrm{lg}$ and $\beta$-lg-fatty acid complexes. (A)-(D) show the results of addition of curcumin to $\beta-\lg , \beta-\lg -\mathrm{C} 8: 0, \beta-\lg -\mathrm{C} 14: 0$, and $\beta-\lg -\mathrm{C} 16: 0$, respectively. $C_{\beta-\text { lg }}=2 \mu \mathrm{M} ; C_{\text {fatty acids }}=300 \mu \mathrm{M} ; C_{\text {curcumin }}=0-50 \mu \mathrm{M}$. where $F$ is the measured fluorescence; $F_{0}$ is the starting fluorescence; $F_{\mathrm{C}}$ is the fluorescence of the fully complexed protein; $K_{\mathrm{d}}$ is the dissociation constant; $[\mathrm{P}]_{\mathrm{t}}$ is the concentration of protein; $[\mathrm{Q}]_{\mathrm{a}}$ is the concentration of added curcumin. The reciprocal of $K_{\mathrm{d}}$ is $K_{\mathrm{a}}$.

The values of binding constants and the non-linear regressions fit based on eqn (3) are shown in Fig. 2. The binding constant of curcumin, $K_{\mathrm{a}}$, is $1.19 \times 10^{4} \mathrm{M}^{-1}$ for $\beta$-lg-curcumin, and the $K_{\mathrm{a}}$ is higher in the ternary complexes, $5.55 \times 10^{4} \mathrm{M}^{-1}$ in $\beta$-lg-C8:0-curcumin, $2.13 \times 10^{4} \mathrm{M}^{-1}$ in $\beta$-lg-C14:0-curcumin, and $2.22 \times 10^{4} \mathrm{M}^{-1}$ in $\beta$-lg-C16:0-curcumin. Therefore, it was evident that fatty acids facilitate the binding of curcumin to $\beta$-lg.

When C14:0 and C16:0 were gradually added into the $\beta-\mathrm{lg}$ solution and $\beta$-lg-curcumin complex, the Trp fluorescence intensities approached a maximum at $10 \mu \mathrm{M}$ fatty acid concentration, and then gradually decreased (Fig. 3). It revealed that when $\beta-\lg$ was saturated with curcumin in advance, C14:0 and C16:0 could still interact with $\beta-\mathrm{lg}$. While the addition of C8:0 had little influence on the fluorescence intensity of $\beta$-lg Trp. Similar observations were seen in previous study which clarified the crystallographic structure of C8:0 binding to $\beta$-lg. ${ }^{29}$ In fact, the results were due to the lower affinity of $\beta$-lg-C8:0 compared to those of other fatty acids with longer chains. It was demonstrated that the binding affinity of fatty acids to $\beta$-lg increased as the hydrocarbon chain lengthened, and the highest binding affinity was observed in $\beta$-lg-C16:0 complex..$^{23,30}$ The main reasons were that the addition of methylene groups in hydrocarbon chain could increase the hydrophobic interactions between fatty acids and $\beta$-lg, and furthermore, $\beta$-lg binding
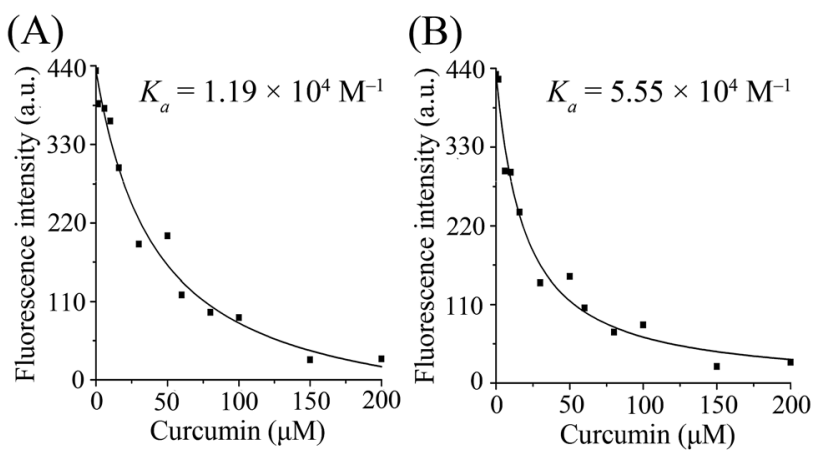

(C)

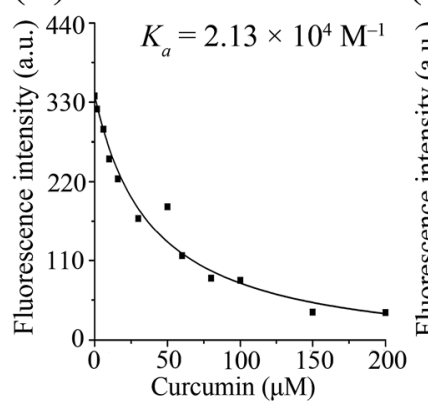

(D)

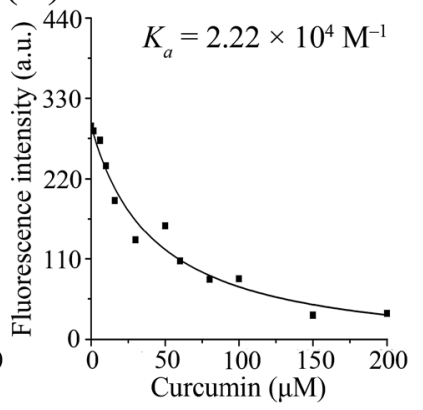

Fig. 2 The non-linear regressions and the binding constants of curcumin bound to $\beta$-lg. (A)-(D) show the regression curves in $\beta$-lg-curcumin, $\beta$-lg-C8:0-curcumin, $\beta$-lg-C14:0-curcumin, and $\beta$ lg-C16:0-curcumin, respectively. $C_{\beta-\lg }=2 \mu \mathrm{M} ; C_{\text {fatty acids }}=300 \mu \mathrm{M}$; $C_{\text {curcumin }}=0,1.6,6,10,16,30,50,60,80,100,150,200 \mu \mathrm{M}$. 


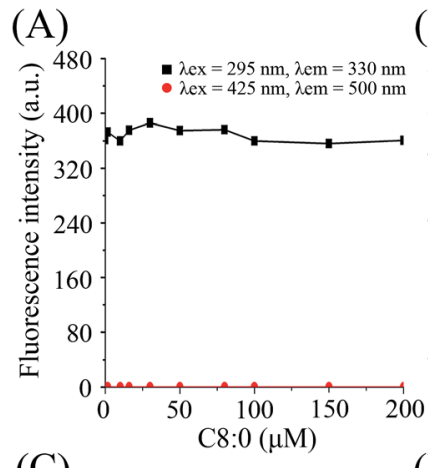

(C)

(B)

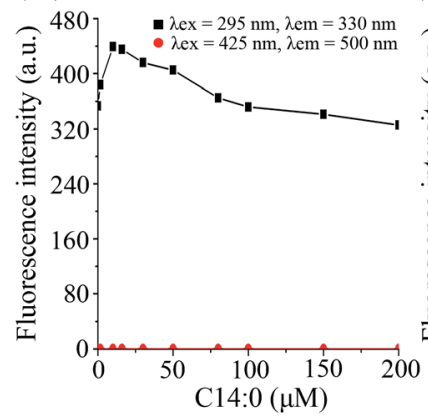

(E)

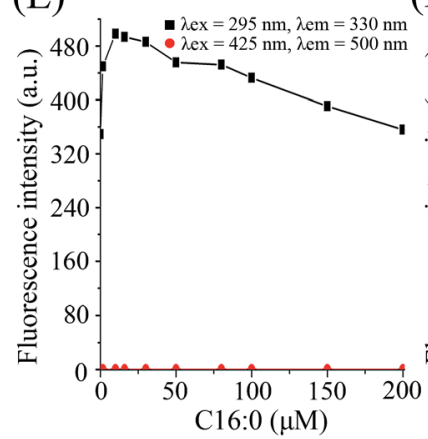

(F)

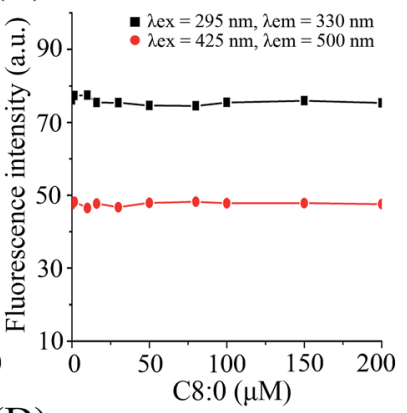

(D)
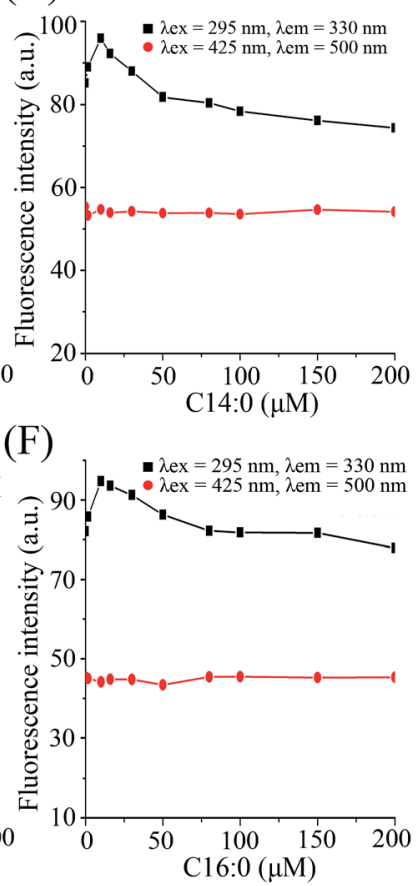

Fig. 3 Fluorescence emission intensity with addition of fatty acids to $\beta$-lg and the $\beta$-lg-curcumin complex. The results were obtained from complexes containing C8:0 (A and B), C14:0 (C and D), and C16:0 (E and $F$ ). $A, C$, and $E$ were recorded in the absence of curcumin; $B, D$, and $F$ were recorded in the presence of curcumin $(150 \mu \mathrm{M}) . C_{\beta-\lg }=2 \mu \mathrm{M}$; $C_{\text {fatty acids }}=0-200 \mu \mathrm{M}$.

pocket for fatty acid accommodated the best with an aliphatic chain constituted by 16 carbon atoms. ${ }^{23}$ Similar results were reported when fatty acids bound to myoglobin. ${ }^{31}$ In addition, the fluorescence of curcumin at $500 \mathrm{~nm}$ showed no significant modification with the additions of fatty acids, which meant that the binding of fatty acids did not induce release of curcumin from the complexes.

\section{CD measurements}

To determine the effect of binding behaviors on the conformation of protein, the conformational transitions of $\beta$-lg in $\beta$-lg-curcumin, $\beta$-lg-fatty acids, and the ternary complexes were monitored by far UV CD spectroscopy (Fig. 4). The far UV CD spectrum showed a broad negative minimum around $217 \mathrm{~nm}$, which was characteristic of $\beta$-sheet structure. After the addition of curcumin and fatty acids, the far UV CD spectra remained similar to that of native $\beta-\mathrm{lg}$, indicating that the binding of ligands to $\beta$-lg did not significantly affect the secondary structure of the protein. Furthermore, the proportions of secondary structures also showed little difference among the samples (Table 1). The effect of ligands binding on secondary structure of protein are various in different binding behaviors. It was reported that the interaction of genistein bound to bovine
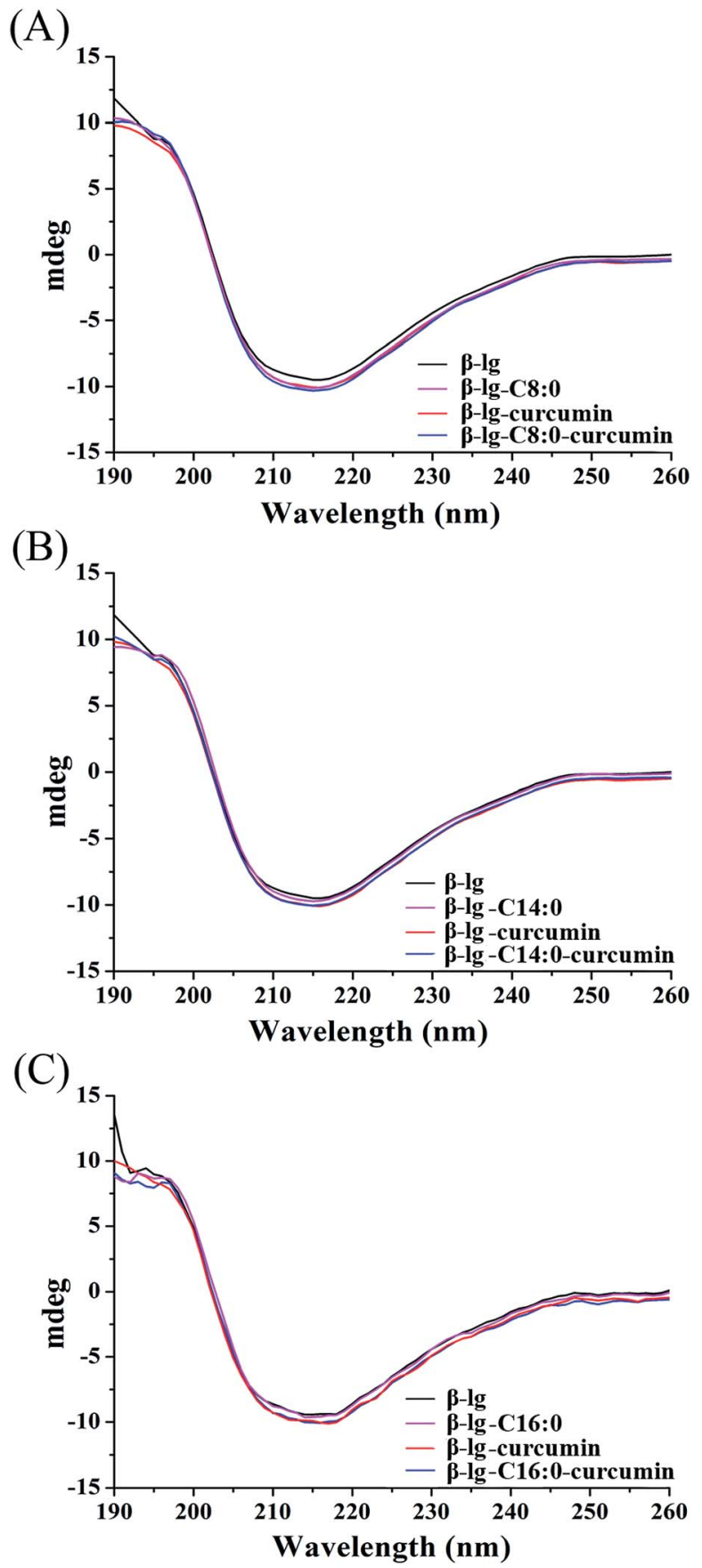

Fig. 4 Far UV spectra of $\beta$-lg and $\beta$-lg-based complexes. The spectra of $\beta$-lg, $\beta$-lg-curcumin, $\beta$-lg-C8:0, and $\beta$-lg-C8:0-curcumin are represented in (A), and the spectra related to $C 14: 0$ and $C 16: 0$ are shown in (B) and (C), respectively. 
serum albumin led to conversion of the $\alpha$-helix to $\beta$-sheet and turn structures due to a partial protein unfolding. ${ }^{4}$ While, in our study, the results were in agreement with several previous studies, which had shown that the interaction of ligands with $\beta$-lg has no significant effect on protein secondary structure. ${ }^{15,32}$ It was suggested that the loops of $\beta$-lg was relatively flexible, and $\beta$-lg did not require extensive changes the structures to allow ligand binding in the calyx..$^{33,34}$

\section{DLS measurements and stability analysis}

The interactions between $\beta$-lg and curcumin or the fatty acids were evaluated in terms of particle sizes and zeta potentials (Table 2), and the sample particle size distribution curves are shown in Fig. S2. $\dagger$ The polydispersity index remained within an acceptable range $(<0.5)$ in all samples (data not shown). The particle sizes and particle size distributions of the $\beta$-lg-fatty acids were close to those of $\beta-\mathrm{lg}$, as the dimeric structures were mostly unaffected by ligand binding. By contrast, the values of mean size increased dramatically with the addition of curcumin (from $6.4 \pm 0.3 \mathrm{~nm}$ to over $110 \mathrm{~nm}$ ). The previous study also found that complexation with curcumin was seen to significantly increase the particle size of soy protein isolate nanoparticles..$^{35}$ It was reported that the addition of curcumin accompanying with ethanol could expose more hydrophobic sites of $\beta-\mathrm{lg}$, and deprived the surface charge. This process caused the formation of larger particles since hydrophobic association increased and electrostatic repulsion reduced. ${ }^{11}$

Table 1 Secondary structure (circular dichroism spectra) of $\beta$-lg and $\beta$-lg-based complexes

\begin{tabular}{lclll}
\hline Samples & $\begin{array}{l}\alpha \text {-Helix } \\
( \pm 1 \%)\end{array}$ & $\begin{array}{l}\beta \text {-Sheet } \\
( \pm 3 \%)\end{array}$ & Turn $( \pm 1 \%)$ & $\begin{array}{l}\text { Unordered } \\
( \pm 2 \%)\end{array}$ \\
\hline$\beta$-lg & 10.4 & 47.5 & 15.4 & 26.7 \\
$\beta$-lg-curcumin & 10.3 & 47.5 & 16.5 & 25.7 \\
$\beta$-lg-C8:0 & 10.4 & 47.1 & 15.3 & 26.1 \\
$\beta$-lg-C14:0 & 10.1 & 48.3 & 15.5 & 25.9 \\
$\beta$-lg-C16:0 & 9.4 & 48.7 & 16.8 & 25.7 \\
$\beta$-lg-C8:0-curcumin & 10.3 & 47.7 & 15.7 & 24.7 \\
$\beta$-lg-C14:0-curcumin & 9.4 & 49.2 & 15.8 & 25.6 \\
$\beta$-lg-C16:0-curcumin & 8.9 & 48.8 & 16.7 & 25.6
\end{tabular}

Table 2 The mean sizes and zeta potentials of the samples ${ }^{a}$

\begin{tabular}{lcl}
\hline Samples & Size $(\mathrm{nm})$ & Zeta potential $(\mathrm{mV})$ \\
\hline$\beta$-lg & $6.4 \pm 0.3 \mathrm{a}$ & $-11.6 \pm 0.2 \mathrm{a}$ \\
$\beta$-lg-curcumin & $113 \pm 2 \mathrm{~b}$ & $-30.4 \pm 0.3 \mathrm{e}$ \\
$\beta$-lg-C8:0 & $6.5 \pm 0.2 \mathrm{a}$ & $-10.5 \pm 0.5 \mathrm{a}$ \\
$\beta$-lg-C14:0 & $6.7 \pm 0.4 \mathrm{a}$ & $-23.5 \pm 0.4 \mathrm{c}$ \\
$\beta$-lg-C16:0 & $6.9 \pm 0.2 \mathrm{a}$ & $-20.1 \pm 0.5 \mathrm{~b}$ \\
$\beta$-lg-C8:0-curcumin & $115 \pm 3 \mathrm{~b}$ & $-25.9 \pm 1.3 \mathrm{~d}$ \\
$\beta$-lg-C14:0-curcumin & $145 \pm 2 \mathrm{c}$ & $-29.1 \pm 0.9 \mathrm{e}$ \\
$\beta$-lg-C16:0-curcumin & $146 \pm 4 \mathrm{c}$ & $-30.8 \pm 1.2 \mathrm{e}$
\end{tabular}

${ }^{a}$ Means with different letters in the same column are significantly different at $p<0.05$.
When C8:0 was added to form ternary complex, the particle size of complex showed little difference. While, with the addition of C14:0 and C16:0, further significant increases in particle sizes compared to $\beta$-lg-curcumin were observed $(p<0.05)$, from $113 \pm$ $2 \mathrm{~nm}$ to $145 \pm 2 \mathrm{~nm}$ and $146 \pm 4 \mathrm{~nm}$, respectively. The reason of the increased particle size in ternary complexes may be related to the aggregation and cross-linking of $\beta$-lg caused by curcumin. The specific mode might be similar to the lactoferrin-EGCG aggregates, in which EGCG acted as linkage among proteins. ${ }^{36}$

The zeta potential results indicated that all samples were negatively charged, and did not exceed absolute values of $40 \mathrm{mV}$, which is the signal of complex stabilization by electrostatic interactions. ${ }^{37}$ Thus, the physical stabilities of the samples could not be explained by electrostatic stabilization, and this suggested that other forces, including hydrophobic interactions, Van der Waals interactions or steric overlap interactions, were involved. Except for C8:0, all ligands significantly increased the negative charges of $\beta-\lg (p<0.05)$, revealing a higher colloidal stability upon their addition. The zeta potentials of the $\beta$-lg-fatty acids (C14:0 and C16:0) were around $-20.0 \mathrm{mV}$, and that of $\beta$-lg-curcumin was $-30.4 \pm 0.3 \mathrm{mV}$. The biggest change was observed between $\beta-\lg (-11.6 \pm 0.2 \mathrm{mV})$ and $\beta$-lg-C16:0-curcumin $(-30.8 \pm 1.2 \mathrm{mV})$.

Moreover, the stability of the ternary complexes were evaluated by DLS measurement and Turbiscan stability analysis. As shown in Fig. S3, $\dagger$ the particle sizes and zeta potentials of the ternary complexes showed little differences during storage time. The Turbiscan curves also observed no destabilization phenomena in the complexes (Fig. S4†). Therefore, the results of both two methods verified that the ternary complexes were stable at $4{ }^{\circ} \mathrm{C}$ for at least 14 days.

\section{Loading efficiencies}

The loading efficiencies of curcumin and fatty acids in their $\beta$-lg complexes are presented in Table 3. Since C8:0 resulted in nearly no changes in fluorescence and dynamic light scattering measurements, the loading efficiencies of fatty acids were evaluated in the complexes including C14:0 and C16:0. The loading efficiency of curcumin in $\beta$-lg-curcumin was $49 \pm 2 \%$, and this significantly increased in the presence of fatty acids $(p<0.05)$. The biggest increase was observed when C14:0 was added, with a loading efficiency of $58 \pm 2 \%$ for $\beta$-lg-C14:0curcumin. In terms of fatty acids, the loading efficiency of

Table 3 Loading efficiencies of curcumin and fatty acids in samples ${ }^{a}$

\begin{tabular}{lll}
\hline Samples & Curcumin (\%) & Fatty acids (\%) \\
\hline$\beta$-lg-curcumin & $49 \pm 2 \mathrm{a}$ & N/A \\
$\beta$-lg-C14:0 & N/A & $46 \pm 1 \mathrm{~b}$ \\
$\beta$-lg-C16:0 & N/A & $47 \pm 2 \mathrm{~b}$ \\
$\beta$-lg-C14:0-curcumin & $58 \pm 2 \mathrm{~b}$ & $32 \pm 2 \mathrm{a}$ \\
$\beta$-lg-C16:0-curcumin & $57 \pm 2 \mathrm{~b}$ & $34 \pm 3 \mathrm{a}$
\end{tabular}

${ }^{a}$ Means with different letters in the same column are significantly different at $p<0.05$. N/A, not applicable. 
C14:0 was $32 \pm 2 \%$ in $\beta$-lg-C14:0-curcumin and that of C16:0 was $34 \pm 3 \%$ in $\beta$-lg-C16:0-curcumin. On the basis of the loading efficiencies of curcumin and fatty acids, it showed that the ternary complexes were able to deliver curcumin and the fatty acids simultaneously, and furthermore, the presence of fatty acids enhanced the loading efficiencies of curcumin in the complexes.

\section{Binding behaviors in the formation of the ternary complexes}

To determine the preferred binding sites on $\beta$-lg, docking experiments were performed with curcumin. When $\beta$-lg interacted with curcumin alone, the results showed that curcumin was surrounded by Phe105, Val41, Lys60, Leu46, Leu54, Ile56, and Val92 with hydrophobic interactions, and Pro38 with hydrogen bonds (Fig. S5 $\dagger$ ). These amino acids were located near the calyx, with Ile, Leu and Val lining the wall of the calyx, and Phe105 in an orientation that was suitable to establish a $\pi-\pi$ interaction with the phenol ring of curcumin. ${ }^{15}$ Thus, the result indicated that curcumin binding to $\beta$-lg in the calyx occurred mainly through hydrophobic interactions. According to a crystallographic study, the primary binding site of C14:0 and C16:0 in $\beta-\lg$ are also the calyx, with end of ligand hydrocarbon chain bound deep in the hydrophobic part of the calyx and carboxyl group located near the calyx entrance. ${ }^{38} \mathrm{With}$ respect to the ternary complexes, when C16:0 occupied the calyx site first, there was another binding site at the surface region for curcumin (Fig. 5). The result for C14:0 was similar to that for C16:0 (data not shown). Curcumin located near Trp19, Tyr20, Thr125, Pro126, Glu158 and Glu44 through both hydrophobic interactions and hydrogen bonds, which was similar to the binding site in $\beta$-lg-resveratrol and $\beta$-lg-isoxazole interactions. ${ }^{39,40}$ Moreover, the binding mode for curcumin out of the $\beta$-lg calyx was still stable when solvation and flexibility was present in the biological system (Fig. S6†). C14:0 and C16:0 filled available spaces in the calyx with the size and shape corresponding well to geometry of the binding site, and Phe105, a key protein residue in the interaction, could specially provide an additional hydrophobic clamp for C16:0. ${ }^{29,38}$ Nevertheless, similar phenomenon was not observed with curcumin and thus might result in the preference for fatty acids appearing in the calyx. While, the presence of C8:0 showed little effects on the binding of curcumin since its hydrocarbon tail is shorter compared to the other fatty acids, and thus could not insert deep in the protein calyx. ${ }^{29}$ Moreover, fatty acid, especially C16:0, is considered to be natural $\beta$-lg ligands with relative high affinity compared with other ligands. ${ }^{38}$ The binding affinities of C14:0 and C16:0 are ranged between $10^{5}-10^{7} \mathrm{M}^{-1},{ }^{30}$ which is much higher than that obtained for curcumin in this study $\left(1.19 \times 10^{4} \mathrm{M}^{-1}\right)$. Therefore, the possible mechanisms involved in the formation of the ternary complexes are shown in Fig. 6. C14:0 and C16:0 were more likely to be located in the calyx preferentially over curcumin, resulting in the curcumin occupied in a different binding site. Certainly, the simulations provide preliminary information, and further studies need to be carried out to unveil the more detailed mechanism.

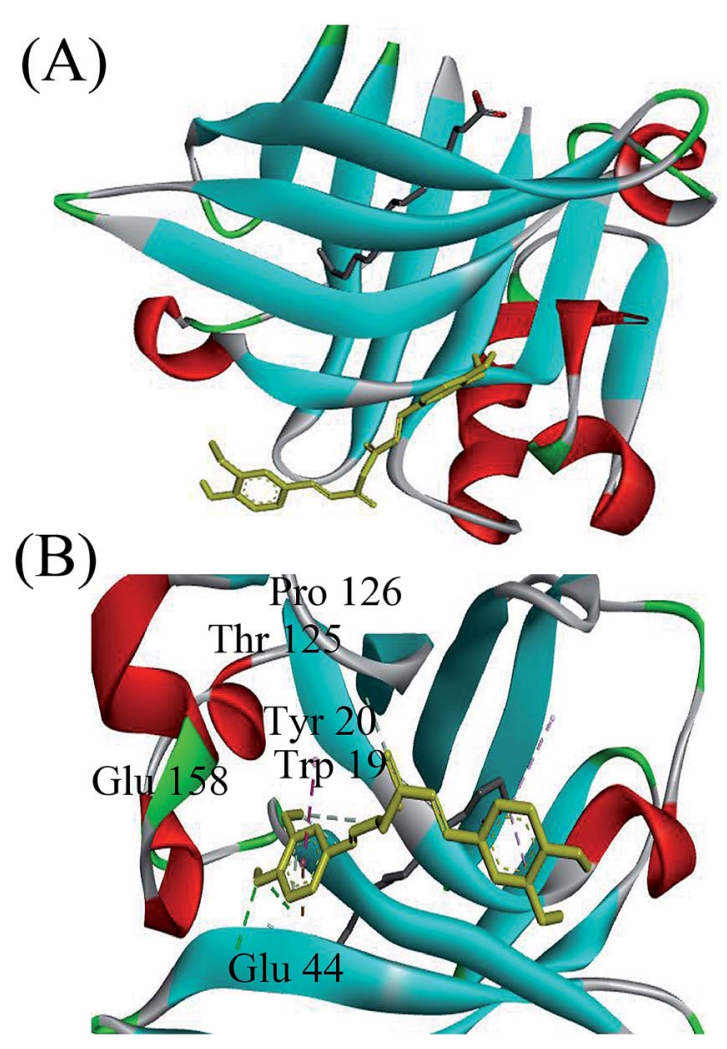

Fig. 5 Docked structure corresponding to the minimum energy conformation for the complex. (A) Overview of binding behaviors of $\beta$-lg with curcumin and C16:0. (B) Detailed illustration of the amino acids near curcumin. In the complex, the yellow ligand represents curcumin, and the grey ligand represents C16:0.

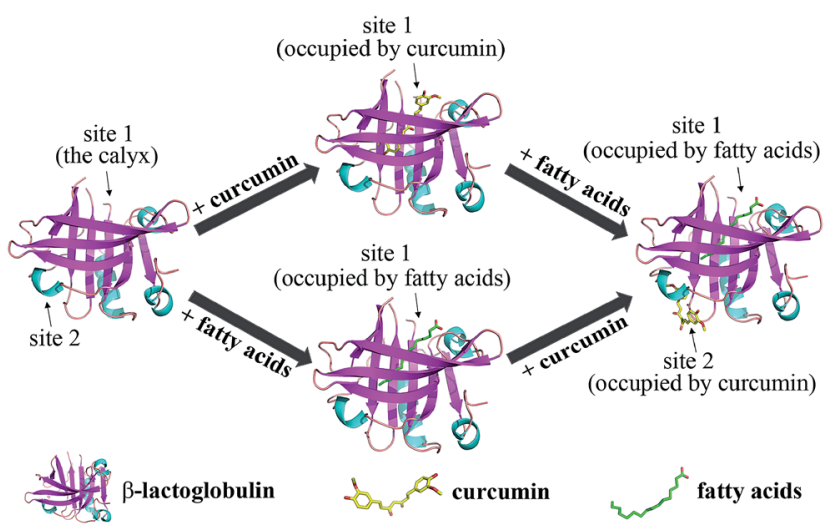

Fig. 6 Schematic representation of the possible mechanisms involved in the formation of the ternary complexes of $\beta$-lg, curcumin, and fatty acids (C14:0 or C16:0).

\section{Conclusions}

Our results showed that $\beta$-lg could interact with curcumin and the fatty acids simultaneously. Compared to the interaction of single ligand with $\beta$-lg, the binding constant of curcumin enhanced. The particle sizes of the ternary complexes showed significant increases, but the secondary structure of $\beta$-lg was 
unchanged. Furthermore, the loading efficiencies of the fatty acids (C14:0 and C16:0) were more than 30\% in the ternary complexes, and those of curcumin were over $50 \%$. Therefore, the results suggest the $\beta$-lg-based nanocarrier could be of great potential to deliver more than one nutraceutical simultaneously in the food industry.

\section{Conflicts of interest}

There are no conflicts of interest to declare.

\section{Acknowledgements}

This work was supported by National Natural Science Foundation of China (Grant No. 31401492), the Ministry of Science and Technology of China (Grant No. 2016YFD0400804), and Chinese Universities Scientific Fund (Grant No. 2017QC086). The funders had no role in the design, analysis or writing of this article. We also thank Dr Mengchi Sun from the Key Laboratory of Structure-Based Drug Design and Discovery, Shenyang Pharmaceutical University for his kind assistance with computational simulation.

\section{References}

1 P. Anand, A. B. Kunnumakkara, R. A. Newman and B. B. Aggarwal, Mol. Pharmaceutics, 2007, 4, 807-818.

2 A. J. Ruby, G. Kuttan, K. D. Babu, K. N. Rajasekharan and R. Kuttan, Cancer Lett., 1995, 94, 79-83.

3 A. L. Cheng, C. H. Hsu, J. K. Lin, M. M. Hsu, Y. F. Ho, T. S. Shen, J. Y. Ko, J. T. Lin, B. R. Lin, M. S. Wu, H. S. Yu, S. H. Jee, G. S. Chen, T. M. Chen, C. A. Chen, M. K. Lai, Y. S. Pu, M. H. Pan, Y. J. Wang, C. C. Tsai and C. Y. Hsieh, Anticancer Res., 2001, 21, 2895-2900.

4 P. Bourassa, C. D. Kanakis, P. Tarantilis, M. G. Pollissiou and H. A. Tajmir-Riahi, J. Phys. Chem. B, 2010, 114, 3348-3354.

5 L. Li, F. S. Braiteh and R. Kurzrock, Cancer, 2005, 104, 1322-1331. 6 A. Sahu, N. Kasoju and U. Bora, Biomacromolecules, 2008, 9, 2905-2912.

7 A. Anitha, V. G. Deepagan, V. V. D. Rani, D. Menon, S. V. Nair and R. Jayakumar, Carbohydr. Polym., 2011, 84, 1158-1164.

8 H. Yu and Q. Huang, Food Chem., 2010, 119, 669-674.

9 B. Y. Qin, M. C. Bewley, L. K. Creamer, H. M. Baker, E. N. Baker and G. B. Jameson, Biochemistry, 1998, 37, 14014-14023.

10 N. P. Aditya, H. Yang, S. Kim and S. Ko, Colloids Surf., B, 2015, 127, 114-121.

11 Z. Teng, R. Xu and Q. Wang, RSC Adv. , 2015, 5, 35138-35154.

12 M. C. Yang, N. C. Chen, C. J. Chen, C. Y. Wu and S. J. T. Mao, FEBS J., 2009, 276, 2251-2265.

13 A. Shpigelman, Y. Shoham, G. Israeli-Lev and Y. D. Livney, Food Hydrocolloids, 2014, 40, 214-224.

14 M. Li, Y. Ma and M. O. Ngadi, Food Chem., 2013, 141, 1504-1511. 15 A. H. Sneharani, J. V. Karakkat, S. A. Singh and A. G. A. Rao, J. Agric. Food Chem., 2010, 58, 11130-11139.
16 C. D. Kanakis, P. A. Tarantilis, M. G. Polissiou and H. A. Tajmir-Riahi, J. Biomol. Struct. Dyn., 2013, 31, 1455-1466. 17 X. Wang, Y. Liu, L. L. He, B. Liu, S. Y. Zhang, X. Ye, J. J. Jing, J. F. Zhang and M. Gao, Food Chem. Toxicol., 2015, 78, 42-51. 18 Y. Wu, H. Cheng, Y. Chen, L. Chen, Z. Fang and L. Liang, J. Agric. Food Chem., 2017, 65, 3019-3030.

19 G. Kontopidis, C. Holt and L. Sawyer, J. Dairy Sci., 2004, 87, 785-796.

20 A. Mensi, Y. Choiset, H. Rabesona, T. Haertle, P. Borel and J. M. Chobert, J. Agric. Food Chem., 2013, 61, 4114-4119.

21 P. Zimet and Y. D. Livney, Food Hydrocolloids, 2009, 23, 1120-1126.

22 M. Bello, J. Biomol. Struct. Dyn., 2016, 34, 1884-1902.

23 D. Frapin, E. Dufour and T. Haertle, J. Protein Chem., 1993, 12, 443-449.

24 M. Narayan and L. J. Berliner, Biochemistry, 1997, 36, 1906-1911. 25 F. Mohammadi, A. K. Bordbar, A. Divsalar, K. Mohammadi and A. A. Saboury, Protein J., 2009, 28, 117-123.

26 M. Pantusa, L. Sportelli and R. Bartucci, Eur. Biophys. J., 2012, 41, 969-977.

27 W. Yang, C. Xu, F. Liu, C. Sun, F. Yuan and Y. Gao, J. Agric. Food Chem., 2015, 63, 5046-5054.

28 A. Shpigelman, Y. Cohen and Y. D. Livney, Food Hydrocolloids, 2012, 29, 57-67.

29 J. Loch, A. Polit, A. Gorecki, P. Bonarek, K. Kurpiewska, M. Dziedzicka-Wasylewska and K. Lewinski, J. Mol. Recognit., 2011, 24, 341-349.

30 S. Le Maux, S. Bouhallab, L. Giblin, A. Brodkorb and T. Croguennec, Dairy Sci. Technol., 2014, 94, 409-426.

31 S. V. Chintapalli, S. Jayanthi, P. L. Mallipeddi, R. K. Gundampati, S. K. T. Krishnaswamy, D. B. van-Rossum, A. Anishkin and S. H. Adams, J. Biol. Chem., 2016, 291, 251133-251143.

32 L. Liang, H. A. Tajmir-Riahi and M. Subirade, Biomacromolecules, 2008, 9, 50-56.

33 M. Bello and E. Garcia-Hernandez, Biopolymers, 2014, 101, 744-757.

34 L. Dominguez-Ramirez, E. D. Moral-Remirez, P. CortesHernandez, M. Garcia-Garibay and J. Jimenez-Guzman, PLoS One, 2013, 8, e79530.

35 F. P. Chen, B. S. Li and C. H. Tang, J. Agric. Food Chem., 2015, 63, 3559-3569.

36 W. Yang, F. Liu, C. Xu, C. Sun, F. Yuan and Y. Gao, J. Agric. Food Chem., 2015, 63, 5035-5045.

37 O. E. Perez, T. David-Birman, E. Kesselman, S. Levi-Tal and U. Lesmes, Food Hydrocolloids, 2014, 38, 40-47.

38 J. I. Loch, A. Polit, P. Bonarek, D. Olszewska, K. Kurpiewska, M. Dziedzicka-Wasylewska and K. Lewinski, Int. J. Biol. Macromol., 2012, 50, 1095-1102.

39 M. Pantusa, R. Bartucci and B. Rizzuti, J. Agric. Food Chem., 2014, 62, 4384-4391.

40 S. Maity, S. Pal, S. Sardar, N. Sepay, H. Parvej, J. Chakraborty and U. C. Halder, RSC Adv., 2016, 6, 112175-112183. 